\title{
Tagungsbericht
}

\section{Arbeit und Geschlecht im Um- bruch der modernen Gesellschaft - Forschung im Dialog}

Die beiden Sektionen Frauen- und Geschlechterforschung und Arbeits- und Industriesoziologie der Deutschen Gesellschaft für Soziologie haben am 28./29. April 2006 zu einer ersten gemeinsamen Tagung in das Landesinstitut Sozialforschungsstelle Dortmund eingeladen. Die Zukunft gesellschaftlicher Arbeit vor dem Hintergrund sich wandelnder Arbeits- und Geschlechterverhältnisse stand im Mittelpunkt der zweitägigen Tagung, an der über 100 WissenschaftlerInnen teilgenommen haben. Ziel war der Dialog zwischen den beiden Sektionen. Zum Einstieg in die drei Forschungsfelder erfolgte jeweils ein Statement aus jeder Sektion.

Dienstleistungen: Interaktive Arbeit, Wissensarbeit und Privatisierung öffentlicher Dienstleistungen

Martin Baethge (SOFI Göttingen) machte den Auftakt mit einem Statement zu „Industrialismus und Familialismus“. Als eine der Nachwirkungen des Industrialismus und seines familialen Modells des männlichen Alleinernährers habe Dienstleistungsforschung noch keine Leistungs- und Arbeitsorganisationsnormen für die verschiedenen Formen von Dienstleistungen entwickelt; zudem fehle eine Definition von Produktivität für die verschiedenen Formen von Dienstleistungen bisher. Hier müsse Dienstleistungsforschung ansetzen, nach Ansicht Baethges aber nicht nur Arbeitsforschung sein, sondern den sozioökonomischen Kontext von Dienstleistungsarbeit berücksichtigen.

Hildegard Maria Nickel (Humboldt-Universität Berlin) hat sich in ihrem Statement mit ,Tertiarisierung, (Markt-)Individualisierung, sozialer Polarisierung - neuen Konfliktlagen im Geschlechterverhältnis" auseinandergesetzt. Sie thematisierte neben den Chancen für Frauen, die durch das Aufbrechen traditioneller Arbeitsmarktstrukturen entstehen, auch die weiter bestehenden Segmentationen - z.B. die Unterrepräsentanz von Frauen im Bereich hochqua- lifizierter, wissensintensiver Dienstleistungen - und neuen sozialen Risiken für Frauen im Erwerbsleben z.B.durch Teilzeitarbeit oder soziale Dienstleistungen als Niedriglohnsektor. Inklusion über Erwerbsarbeit könne nur gelingen über eine Neubewertung von Feldern wie z.B. Wissens- oder Sorgearbeit. Die auf das Individuum zurückverlagerte Durchsetzung von Gleichberechtigung berge auch die Gefahr einer Polarisierung unter den Frauen.

Die beiden Vorträge und nachfolgenden Diskussionen zeigten, dass gerade Dienstleistungen das Feld sind, an welchem die hohe Bedeutung der Verschränkung von Erwerbsarbeit und Geschlechterarrangements besonders deutlich wird.

Nach diesem diskursiven Auftakt folgte der erste Fachvortrag von Hedwig Rudolph (Wissenschaftszentrum Berlin) über „Geschlechterhierarchische Konturen der Projektarbeit in Unternehmensberatungen“. Sie stellte Ergebnisse einer Studie zu Unternehmensberatungen in Deutschland vor. Die Sicherung der Definitionsmacht gegenüber den Kunden geht in den Beratungsunternehmen einher mit der Etablierung einer männlichen Kultur der Elite. Der formal propagierten Ansicht, Geschlechtergleichheit sei verwirklicht, stehen u.a. der geringere Anteil an Frauen (25\%), ausgeprägte vertikale und horizontale Segregationen und ein ausgeprägter, gender subtext' in Form verdeckter, machtbasierter Arrrangements (Forderung nach unbegrenzter Zeit/Verfügbarkeit, informelle Netzwerke und Leistungsinszenierung) gegenüber.

In der Diskussion wurde vor allem die Verknüpfung dieses gerade in dieser Branche besonders gut sichtbar werdenden ,neoliberalen Paradigmas“ mit konservativen Geschlechtermodellen und die lange vorherrschende männliche Kultur im Management lebhaft diskutiert.

Wolfgang Dunkel (ISF München) und Margit Weihrich (Universität München) machten einen handlungstheoretischen Vorschlag zur Verbindung von Geschlechter- und Arbeitssoziologie und verknüpften Interaktion, Geschlecht und Arbeit in ihrem Vortrag „Doing Gender in der interaktiven Arbeit“. Am Beispiel der Friseurdienstleistungen entwickelten sie ihr Konzept des „working gender“ und gingen der Frage nach, wie Geschlecht zur Lösung der 
Abstimmungsprobleme bei interaktiver (Dienstleistungs-)Arbeit genutzt wird. Die drei Dimensionen "gendering in work", „,working with gender" und "working on gender" böten sich zur Erklärung des strategischen Einsatzes von Geschlecht im Prozess der Dienstleistungserbringung an.

In der Diskussion wurde die Notwendigkeit weiterer Forschung zur Bedeutung von Gender für die Qualität von Dienstleistungen und Art und Weise deren Erbringung hervorgehoben.

Im abschließenden Beitrag zum ersten Dialogfeld analysierte Birgit Riegraf (Universität Bielefeld) die Konzepte des New Public Management unter der geschlechtsspezifischen Perspektive. Als Referenzbeispiel diente ihr dabei Neuseeland. Bei der Umsetzung radikaler Konzepte des New Public Managements und der Neudefinition öffentlicher Aufgaben durch Bezug auf rational-choice-Modelle bestehe die Gefahr einer Retraditionalisierung der Geschlechterverhältnisse, z.B. durch Privatisierung zentraler Bereiche der Daseinsfürsorge, durch Rückverlagerung von Reproduktions-/Careaufgaben in die Familien, Kirchen, Non-Profit-Organisationen, durch Ausdünnung der mittleren Managementebenen in der Verwaltung und durch die Einstellung bisheriger öffentlicher Gleichstellungsmaßnahmen.

Da jedoch auch die Ausgrenzungseffekte des traditionellen bürokratischen Systems nicht geleugnet werden könnten, stellte sich vor allem auch in der Diskussion die Frage, ob und inwiefern gemäßigtere Varianten des NPM, z.B. durch genau definierte Qualitätsstandards oder eine Neutarierung des Verhältnisses Staat/Markt und Zivilgesellschaft, tatsächlich Chancen zum Aufbrechen traditioneller Geschlechterarrangements bieten könnten.

Arbeiten in der globalen Ökonomie und in den Verhältnissen von ,Gender, Race and Class'

Ilse Lenz (Universität Bochum) setzte sich in ihrem Statement „Die Globalisierung der Arbeit und die Rekonfigurationen von Geschlecht, Klasse, Kultur" mit transnationalen Unternehmen und deren Auswirkungen auf nationale und betriebliche Geschlechterarrangements und -ungleichheiten auseinander. Am Beispiel der Entwicklungen u.a. in Malaysia und Südkorea verdeutlichte sie das Zusammenwirken lokaler Gender- und Arbeitskultur mit dem im Unternehmen vorherrschenden Produktionsparadigma und nationalen/internationalen Politikvorgaben. Gerade transnationale Unternehmen übernehmen in der Regel nicht die regionale Arbeitskultur, es entstehen in den Unternehmen neue Segmentationslinien, z.B. nach Ethnizität, Geschlecht und/oder der Unterscheidung Stammbelegschaft/Randbelegschaft.
Boy Lüthje (Universität Frankfurt) ergänzte die Thematik in seinem Vortrag „,Desorganisierter Despotismus'. Globale Produktion, Arbeitsmigration und geschlechtliche Diskriminierung in China" um den Aspekt der „netzwerkbasierten Massenproduktion“. Vor allem in der IT-Branche führten derart komplexe, weltweit verteilte Produktionssysteme zu einer Segmentierung/Taylorisierung der Produktion auf höchstem technischen Niveau und gingen einher mit geschlechtlicher und sozialer Diskriminierung als einem zentralen Konstitutionsmoment der „Kernbelegschaften".

Brigitte Schulze (Universität Frankfurt) beschrieb, wie sich marginalisierte Frauen, ,jenseits von kapitalistisch funktionaler Arbeit erleben, erinnern, erzählen, erträumen“. Im Rahmen eines Videofilmprojektes hat sie dieArbeits-/Lebens- und Erfahrungsräume indischer Dalit-Frauen (die „Unberührbaren“) aufgegriffen. Sie plädierte dafür, die sich meist nur um mehrwertschaffende Arbeit drehenden Diskurse um den Aspekt der Subsistenzarbeit zu ergänzen.

Karrieren, Lebensentwürfe und Arbeitsbiographien im Wandel der Arbeits- und Geschlechterarrangements

In seinem Statement zur ,Subjektivierung der Arbeit und Subjektivierung von Arbeitskraft" widmete sich Günter Voß (TU Chemnitz) dem Idealtypus des Arbeitskraftunternehmers. Selbst-Kontrolle, Selbst-Ökonomisierung und Selbst-Rationalisierung seien an sich zwar genderneutral gedacht, deren Erscheinungen jedoch sozial different und geschlechtspezifisch konnotiert. Zugespitzt mündet er in der Frage, ob Frauen andere Subjektqualititäten hätten, die sie besonders geeignet machten für den Arbeitskraftunternehmer.

Sylvia Wilz (FernUniversität Hagen) erinnerte in ihrem Statement zu „De-Institutionalisierung, Individualisierung und Personalisierung?" daran, dass die Frage, inwieweit Geschlecht vor dem Hintergrund sich wandelnder Arbeitsarrangements tatsächlich als Zuweisungskriterium an Bedeutung verliere, auf der Basis vorliegender Untersuchungen nicht beantwortet werden könne. Zwar könnte rein auf der Basis von Geschlecht keine Berufskarriere per se ausgeschlossen werden, statistisch seien Geschlechterungleichheiten aber nach wie vor wirksam. Statt sich zu sehr an einem Idealtypus wie dem Arbeitskraftunternehmer zu orientieren, schlug sie drei Analyseebenen vor: die Beziehung zwischen Person und Organisation, zwischen Person und Markt und der Person zu sich selbst bzw. den gesellschaftlichen Strukturierungsprinzipien. 
Auch in der Debatte wurde vor allem die Frage der Handhabbarkeit des Modells des Arbeitskraftunternehmers für die empirische Forschungspraxis kritisch diskutiert. Die doppeldeutige Verwendung des Begriffes der Subjektivierung wurde problematisiert, welcher sowohl negativ als Verschärfung von Entfremdung als auch positiv im Sinne der Selbstbestimmung der Arbeit gebraucht werde.

Stefanie Ernst (Universität Hamburg) thematisierte in ihrem Vortrag,,Verdichtung, Flexibilisierung und Selbstvermarktung" Fremd- und Selbstzwänge in hochqualifizierten, prekären Beschäftigungsfeldern und die jeweiligen Bewältigungsstrategien der Individuen. Im Vortrag und der anschließenden Diskussion wurden die Vorzüge debattiert, die Figurationssoziologie N. Elias' zu nutzen, um dem Begriffder Subjektivierung den der Selbstregulierung entgegenzusetzen.

Basierend auf einer Studie zu Erwerbsarbeit und dem Einfluss von Geschlechtlichkeit aufErwerbsbiographien stellte Ute Luise Fischer (Universität Dortmund) in ihrem Vortrag „Krise der Arbeit, Krise der Sinnstiftung"dieUnterschiede der Lebensgestaltung von Männern und Frauen in den Mittelpunkt ihrer Überlegungen. Die Arbeit als Quelle für Einkommen und Lebenssinn gerate im Zuge der Erosion des Normalarbeitsverhältnisses und der steigenden Arbeitslosigkeit unter Druck. Geschlechterarrangements und bestehende gesellschaftliche Anerkennungsgefüge wandeln sich, allerdings fehlt es nach wie vor an gesellschaftlicher Anerkennung für nicht marktförmig organisierte Arbeit. Der ökonomische Druck führe teilweise sogar zu einer Renaissance des „Ernährermodells“.

Michael Frey und Almut Kirschbaum (Humboldt-Universität Berlin) veranschaulichten am Bespiel des Deutsche Bahn-Konzerns die möglichen Folgen von Vermarktlichung und Subjektivierung für die Beschäftigten. Der Wandel betrieblicher Geschlechterverhältnisse durch Vermarktlichung und Subjektivierung von Arbeit biete riskante Chancen der geschlechterpolitischen Gestaltung. Bei der Deutschen Bahn lasse sich seit der Privatisierung im Prinzip eine De-Thematisierung von Geschlecht und eine Individualisierung der Durchsetzung von Egalitätsansprüchen feststellen. Es eröffneten sich neue Chancen für die Synchronisation von Arbeit und Leben durch die Möglichkeit des Aushandelns individualisierter Commitments, die allerdings in hohem Maße von der individuellen Verhandlungsmacht der Leistungsträgerinnen abhängig seien. Insbesondere diese These des noch am Beginn stehenden Projektes - die Vermarktlichung und das Subjekt als Ansatzpunkte und Chance zur Ermächtigung der Subjekte durch Stärkung von Selbstbefähigung und Selbstver- tretungskompetenz - wurde in der anschließenden Diskussion aufgegriffen. Infrage gestellt wurde, ob sich Vereinbarkeitswünsche wirklich individuell austragen ließen bei gleichzeitiger De-Thematisierung von Geschlecht.

Nach Impulsreferaten von Regina BeckerSchmidt (Universität Hannover), Monika Goldmann (Sozialforschungsstelle Dortmund), Dieter Sauer (ISF München), Klaus Dörre (Friedrich-SchillerUniversität Jena), Ingrid Kurz-Scherf(Philipps-Universität Marburg) entspann sich in der abschließenden Podiumsdiskussion eine lebhafte Diskussion zur „Zukunft der Arbeitsgesellschaft" zwischen den Referent/innen und dem Publikum, in welcher sich querschnittsartig die wichtigsten Argumentationslinien dieser spannenden und anregenden Tagung widerspiegelten: Weitgehend einig war man sich darüber, dass Verschiebungen in den Sphären des kapitalistischen Systems traditionelle Geschlechterhierarchien in Bewegung bringen. Die Möglichkeit der Inanspruchnahme von Subjektivitätspotenzialen durch Frauen blieb allerdings ein eifrig diskutiertes Thema. Auch die Rückkehr sozialer Ungleichheit durch prekäre Beschäftigung und in diesem $\mathrm{Zu}$ sammenhang das Infragestellen von traditionellen - Männern und Frauen zur Verfügung stehenden - Identitätskernen (Entweiblichung und Zwangsfeminisierung) machten deutlich, dass Arbeitsforschung die gesamte Lebens- und Arbeitswelt berücksichtigen muss und sich hier z.B. nicht nur auf die Vereinbarkeitsproblematik beschränken darf. Androzentrische Denkfiguren wie z.B. der männliche Facharbeiter als Vorlage für die Subjektivierungsthese oder der Normalverdiener als Vorlage für die Prekarisierungsthese sind hier eher ein Hindernis. Angesichts derart vielschichtiger gesellschaftlicher Wandlungsprozesse ist mit Blick auch auf die EU-Ebene und den von dort wirkenden Regulierungen die Frage, inwiefern sich die Aufgaben der Gleichstellungspolitik verändern und welche Gefahren eine derart ökonomisch konnotierte Gleichstellungspolitik mit sich bringt.

Die während der gesamten Tagung sehr produktive Diskussion bot die Möglichkeit, Einblicke in den jeweils anderen Forschungsstrang gewinnen zu können. Es gelang erfolgreich Querschnittsthemen zu identifizieren und die Notwendigkeit der inhaltlichen Verknüpfung zukünftiger Debatten und Forschung in den beiden Forschungsrichtungen, über das bisher erfolgte Maß hinaus, zu unterstreichen. Die Beiträge der Tagung werden Anfang nächsten Jahres beim VS Verlag für Sozialwissenschaften veröffentlicht.

Melanie Roski (Dortmund) 